\title{
ÉDITORIAL
}

\section{Recommandations pour la pratique clinique, Nice, Saint-Paul-de-Vence : et de trois !}

\author{
J. Gligorov ${ }^{1}$, M. Namer ${ }^{2}$ \\ ${ }^{1}$ Service d'oncologie médicale, AP-HP, hôpital Tenon, 4, rue de la Chine, F-75970 Paris, France \\ ${ }^{2}$ Centre Azureen de cancérologie, 1, place du Docteur-J.-L.-Broquerie, F-06250 Mougins, France \\ Correspondance : joseph.gligorov@tnn.aphp.fr
}

Les voici ! Beaucoup en ont parlé, discuté et les ont attendues : elles sont maintenant à votre disposition après un long travail de relecture qui, je l'espère, répondra à vos attentes.

Nous avions opté, lors de cette troisième édition, pour cinq thèmes et vous n'en retrouverez que quatre. Comme lors de la première édition des RPC, un des groupes de travail, malgré une revue de la littérature complète, a considéré qu'il était impossible, voire dangereux de répondre à la définition d'une population dite de "bon pronostic ". Et pourtant, me direz-vous, il s'agit d'un problème quotidien. Exact, mais à la décharge de nos experts, la notion la plus importante à intégrer est celle du temps et de la durée. La philosophe Simone Weil écrivait : " La vie elle-même change tellement vite. C'est tellement difficile aujourd'hui de faire des pronostics sur ce que seront les choses dans dix ans ". En effet, la question du pronostic ne peut se concevoir qu'en fonction du recul nécessaire pour admettre la guérison ou un taux de rechute " acceptable ". Le constat principal a donc été, avant même d'aller plus loin, de devoir s'accorder sur la définition du " bon pronostic "; pour citer cet autre philosophe qu'est Bertrand Russel : "dans toute discussion, le plus délicat est toujours de faire la différence entre une querelle de mots et une querelle de fond ". II reste qu'en pratique clinique, les acteurs de soins et de décisions sont de plus en plus désireux de traiter ou d'être traités au plus juste. Cela explique, notamment dans les pays où le dépistage amène son lot considérable de petites tumeurs sans atteinte ganglionnaire, l'importance émergente de nouveaux outils pronostiques, certes en cours de validation, mais déjà utilisables et intégrés dans certains référentiels [1].

Les autres RPC ont porté sur le cancer du sein métastatique, le cancer du sein de la femme non ménopausée, le cancer du sein de la femme âgée et la surveillance. Au moment où ces recommandations paraissent, quelques articles récents viennent conforter ces choix.

En ce qui concerne la prise en charge des populations âgées, on retiendra un article récent des collègues allemands soulignant le sous-traitement des populations âgées par rapport aux standards [3]. Doit-on réaliser un traitement à " l'économie " pour une patiente de 70 ans sans comorbidités importantes qui avait théoriquement, avant le diagnostic, une espérance de vie de plus de dix ans?

Dans le domaine du cancer du sein métastatique, les avancées en termes de développement des traitements sont si importantes qu'on ne peut exclure une réflexion suivie quant à l'intégration des nouveaux traitements (systémiques ou locorégionaux) afin de poursuivre l'objectif principal dans cette situation : augmenter la durée de vie avec une meilleure qualité, mais également définir les interrogations et les voies de recherche futures [2].

Pour la surveillance, on doit en revanche reconnaître un certain désert de publications depuis maintenant plus de 15 ans, amenant peut-être à s'interroger sur l'importance d'une surveillance " à la carte " en fonction du risque lié au stade, à la biologie de la tumeur, mais également de l'impact des traitements.

Encore du travail donc pour la suite. Les RPC 2009 nous auront également permis de construire des ponts collaboratifs avec nos collègues de I'AGO, du NCCN, de Saint-Gallen, 
afin d'aller peut-être, pour les prochaines moutures, vers des recommandations internationales communes concernant les questions urgentes auxquelles nous souhaitons répondre.

Nous vous livrons donc ce travail d'experts représentant un volume non négligeable d'information et de bibliographie toujours dans un seul objectif : améliorer les pratiques pour améliorer le pronostic des patientes prises en charge.

À bientôt donc et bonne lecture !

\section{Références}

1. Goldhirsch A, Ingle JN, Gelber RD, et al. (2009) Thresholds for therapies: highlights of the Saint-Gallen International Expert Consensus on the primary therapy of early breast cancer 2009. Ann Oncol 20(8): 1319-29

2. Gotay CC, Lipscomb J, Snyder CF (2005) Reflections on findings of the Cancer Outcomes Measurement Working Group: moving to the next phase. J Natl Cancer Inst 97(21): 1568-74

3. Hancke K, Denkinger MD, König J, et al. (2009) Standard treatment of female patients with breast cancer decreases substantially for women aged 70 years and older: a German clinical cohort study. Ann Oncol [Epub ahead of print] 